\title{
SOLVENTES DE COLA: ABUSO E EFEITOS NOCIVOS À SAÚDE
}

\author{
Maria de Fátima Menezes Pedrozo* \\ Maria Elisa Pereira Bastos de Siqueira**
}

PEDROZO, M. de F.M. \& SIQUEIRA, M.E.P.B. de. Solventes de cola: abuso e efeitos nocivos à saúde.

Rev. Saúde públ., S. Paulo, 23:336-40, 1989.

RESUMO: $O$ abuso de solventes de cola no Brasil constitui preocupação crescente frente ao aumento do número de usuários, na maioria crianças e adolescentes de baixo nivel sócio-econômico. Objetivou-se apresentar alguns aspectos relativos a este abuso, com ênfase especial aos efeitos nocivos decorrentes da inalação aguda e/ou crônica dos solventes das colas.

DESCRTTORES: Abuso de substâncias, incidência. Solventes, envenenamento.

\section{INTRODUÇÃO}

A prática de inalar os vapores de substâncias químicas que alteram o comportamento e/ou o humor remonta à época dos oráculos gregos, quando eram utilizadas para intensificar experiências religiosas ou místicas. Desde então, muitas substâncias tais como gases anestésicos, propelentes de aerosóis e vários solventes têm sido usados pelo homem.

$\mathrm{Na}$ época atual, o abuso de solventes constitui um sério problema nas sociedades de diversos paises, entre eles o Brasil, envolvendo, principalmente, os adolescentes $\$, 5,7,8,9,12,25,33$.

O primeiro caso, relatado na literatura, de inalação de solventes presentes nas colas, data de 1959 e refere-se a adolescentes americanos. Nos anos 60 este tipo de abuso tomou proporções epidêmicas nos Estados Unidos; na década seguinte constatou-se o envolvimento de crianças e adolescentes de várias partes do mundo, como Canadá, México, Europa, América do Sul, Japão e África do Sul20,26,36,37,38.

Dentre os diferentes produtos químicos com componentes voláteis utilizados - colas, laquê, removedores de esmalte, produtos para limpeza doméstica, "thinner" - as colas são os mais empregados, principalmente, pela população jovem de baixo nível sócio-econômico (glue sniffing) 8,36 .

No Brasil, Carlini-Contrim e Carlini? (1987) realizaram um estudo na cidade de São Paulo, sobre o consumo de solventes e outras drogas, entre 1.836 estudantes de $1^{\circ}$ e $2{ }^{\circ}$ graus, de baixa renda e de idades variando entre 9 e 18 anos. $\mathrm{O}$ uso de solventes de cola foi detectado em $25,2 \%$ dos usuários, após o uso do lançaperfume $(35,9 \%)$, da acetona $(33,8 \%)$, da gasolina
$(31,9 \%)$ e do esmalte $(30,3 \%)$. Estudando uma população diversa - 120 "meninos de rua" e 46 menores internados em comunidades terapêuticas por distúrbio de conduta - os mesmos autores encontraram um quadro diferente de uso de solventes. O uso de cola foi detectado entre $86 \%$ dos "meninos de rua" e $87,5 \%$ dos internados sendo, portanto, o mais utilizado inalante seguido, respectivamente, nos dois grupos estudados, pelo lançaperfume $(43,0 \mathrm{e}$ $43,7 \%)$, benzina $(32,3$ e $37,5 \%)$, esmalte $(44,1$ e $6,3 \%$ ) e acetona $(4,3$ e $31,2 \%)$.

Um levantamento por nós realizado no livro de Registro da Seção de Voláteis do Serviço Técnico de Toxicologia Forense do Instituto Médico Legal (IML) de São Paulo demonstrou, no período de 1982 à 1987 , um crescente número de casos apreendidos de abuso de colas (Fig.).

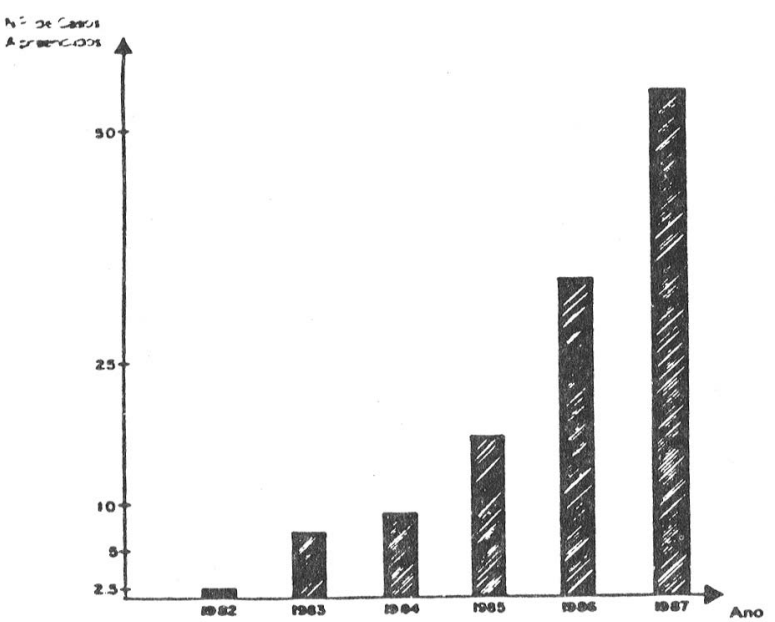

Figura - Casos apreendidos de abuso de solventes de cola na cidade de São Paulo, no período de 1982 1987.

* Instituto Médico Legal do Estado de São Paulo - Rua Teodoro Sampaio, 151 - 05405 - São Paulo, SP - Brasil.

** Departamento de Fisiologia e Farmacologia da Escola de Farmácia e Odontologia de Alfenas - Rua Gabriel Monteiro da Silva, 714 - 37130 - Alfenas, MG - Brasil. 
De fato, o número de casos apreendidos e registrados refletem apenas a tendência do aumento desse tipo de abuso, sendo desconhecido o núm'ero real de usuários, uma vez que a maioria permanece não identificada.

\section{PERFIL DO USUÁRIO E FORMAS DE USO}

O abuso de solventes é quase que um fenômeno exclusivo de adolescentes. As estatísticas mostram que a idade média do usuário vem diminuindo paulatinamente com o tempo, existindo relatos de casos com crianças de 5 a 7 anos $3,36,37$.

Em geral, os adolescentes são provenientes de famílias numerosas e de baixo nível sócioeconômico, nas quais o relacionamento familiar é insatisfatório (pais separados ou envolvidos com drogas e/ou outras atividades ilícitas). Além desses fatores predisponentes contribuem os relativos ao período da adolescência: mudanças físicas e emocionais que tornam o indivíduo inseguro, ansioso, mal humorado, imprevisível e vulnerável2,21,30.

São ainda fatores determinantes da dependência: a influência do grupo de amigos, o baixo custo, a facilidade de aquisição (venda livre) e 'os efeitos euforizantes obtidos pela inalação dos solventes que reforçam o uso repetido $14,17,27,28$.

$\mathrm{O}$ abuso de inalantes não se restringe, entretanto, aos adolescentes. Lowestein (1985) ${ }^{25}$, em estudo realizado na cidade de Londres, demonstrou que a inalação de solventes por indivíduos de idade entre 17 e 33 anos é um hábito mais difundido do que o esperado, sendo muitos deles trabalhadores expostos, ocupacionalmente, aos solventes.

A inalação de solventes presentes nas colas é usualmente realizada em grupo. $\mathrm{O}$ modo mais difundido de uso é a inalação de vapores da cola depositada em sacos plásticos, o que diminui sua dispersão, aumentando seus efeitos. Estes, quando euforizantes, também podem ser conseguidos através da inalação direta de vapores da própria embalagem, com ou sem aquecimento prévio da mesma ${ }^{18}$.

Dentre os produtos mais utilizados, destacam-se as colas para aeromodelismo, material plástico e sapato. Nessas colas os principais solventes são os hidrocarbonetos aromáticos como o tolueno e os alifáticos como o n-hexano, além de cetonas alifáticas como as alquilcetonas. Em São Paulo, a cola para sapatos "Cascola", que contém o tolueno e o n-hexano como principais solventes, foi o principal produto apreendido pelo Instituto Médico Legal.
Os efeitos euforizantes e tóxicos decorrentes do hábito de usar inalantes dependem da intensidade e frequiência das inalações, assim como, do estado emocional do usuário antes desta exposição ${ }^{2,24}$. Inicialmente, $O$ indivíduo apresenta euforia semelhante a provocada pelo álcool, seguida de confusão mental, desinibição e alucinações visuais e/ou auditivas. Tais efeitos podem ter duração de 3 a 60 minutos 2,4,9.

A frequiência do uso (número de sessões por dia e/ou semanas) é variável, de uma vez por semana até 24 vezes ao dia, podendo se prolongar por vários anosi8.

Alguns autores acreditam que o abuso de inalantes se restringe à adolescência 14,15,16,24,29,36,38. Parece existir relação entre a inalação de solventes presentes em cólas (glue-sniffing) e o abuso simultâneo de outras drogas como o álcool, a maconha e a cocaína2, embora estudos visando corroborar essa teoria tenham sido inconclusivos ${ }^{18}$.

\section{PRINCIPAIS EFEITOS NOCIVOS}

\section{Em Nivel do Sistema Nervoso Central}

Os solventes orgânicos, de modo geral, são depressores do sistema nervoso central e, de acordo com o período, freqüência e intensidade da exposição, provocam desde sonolência, confusão mental e cefaléia, até depressão respiratória, coma e morte.

As cetonas promovem narcose menos intensa que o tolueno e o n-hexano, ainda que de maior duração, provavelmente, por apresentarem lipossolubilidade e velocidade de eliminação menores ${ }^{6,22}$. Assim, também, podem levar à depressão do Sistema Nervoso Central (SNC) com falência cardiorespiratória e coma.

Os vários estudos, realizados com voluntários de diferentes faixas etárias que faziam uso por tempo prolongado de produtos contendo tolueno, demonstraram alterações em nível do SNC, caracterizadas por distúrbios neurológicos e comportamentais: perturbações das funções cognitivas, da atenção, da memória visual e verbal além de epilepsia idiopática, encefalopatia permanente acompanhada de atrofia cerebral e cerebelar 1,10,14,15,16,17,19,25,32.

Também foi observado neuropatia periférica, iniciando com parestesia das extremidades atribuidas à intoxicação por estes solventes. Estas alterações surgem em menos de um ano após o início da exposição continuada e tendem a evoluir, mesmo após a interrupção da inalação, para distúrbios graves como a tetraplegia 19. 
As polineuropatias, também atribuídas ao tolueno, não têm seu mecanismo de ação bem elucidado. Para compostos como o n-hexano e a metil n-butilcetona é provável que tal efeito se deva ao seu produto de biotransformação comum, a 2,5-hexanodiona. Sabe-se que esse metabólito interage com componentes dos axônios, podendo causar degeneração nervosa central e periférica $11,13,34$.

Entretanto, é temeroso atribuir determinado efeito a um solvente específico uma vez que, nos produtos utilizados, estão presentes vários compostos voláteis que provocam efeitos similares.

\section{Em Nivel de Outros Tecidos e Órgãos}

Outros tecidos do organismo podem ser também afetados pelos solventes, tais como a pele, mucosas, tecido hematopoiético, fígado e rins.

A exposição por curto prazo ao n-hexano e as cetonas provoca irritação das mucosas oculares e das vias aéreas superiores ${ }^{23}$, ao passo que a exposição por longo prazo leva a laringite, gastralgia, dermatose e sinais cutâneos característi$\cos$ da ação destrutiva do $n$-hexano sobre a pele ${ }^{18}$.

O tolueno atua também em nível muscular promovendo, devido ao desiquilíbrio eletrolítico por ele induzido (hipocalemia, hipofosfatemia), provocando fraqueza muscular generalizada $16,19,31,32$.

A ação tóxica do tolueno sobre o fígado pode levar à hepatite e hepatomegalia. Entretanto, até o momento, não se tem dados que permitam concluir sobre a irreversibilidade ou não desses efeitos hepatotóxicos.

A nivel renal já foi comprovado hematúria, acidose tubular e síndrome de Fanconi (glicosúria e fosfatúria) devido à inalação crônica de tolueno 14,22,26.

As alterações hematológicas - discrasias sangüíneas, eosinofilia, anemia, leucopenia -, observadas na intoxicação a longo prazo por tolueno, são atribuidas ao benzeno presente como impureza neste produto 19,35 .

\section{SINTOMATOLOGIA}

Devido ao abuso de solventes, que apresentam principalmente neurotoxidade, um conjunto de sinais e sintomas decorrentes da intoxicação aguda e/ou crônica pode ser observado.

A evolução da ação de solventes sobre o SNC em exposição de curta duração pode ser resumida como na Tabela 1.
TABELA 1

Evolução de sinais e sintomas decorrentes da inalação de solventes sobre o Sistema Nervoso Central.*

\begin{tabular}{|c|c|}
\hline Fases & Sinais e Sintomas \\
\hline 1. Fase: Excitação & $\begin{array}{l}\text { euforia, tontura; alucinaçōes vi- } \\
\text { suais e auditivas; tosse, saliva- } \\
\text { çăo, fotofobia; náuseas, vômi- } \\
\text { tos; comportamento bizarro. }\end{array}$ \\
\hline \multicolumn{2}{|l|}{ 2! Fase: Depressão } \\
\hline a) leve & $\begin{array}{l}\text { confusão, desorientação, perda } \\
\text { do au tocontrole; diplopia, visão } \\
\text { turva, tinnitus; cefaléia; analgè- } \\
\text { sia, palidez. }\end{array}$ \\
\hline b) moderada & $\begin{array}{l}\text { sonolência, ansiedade; ataxia e } \\
\text { incoordenação muscular; dimi- } \\
\text { nuição dos reflexos; nistagmo }\end{array}$ \\
\hline c) intensa & $\begin{array}{l}\text { inconsciência; delírio, estupor; } \\
\text { sonhos bizarros; convulsōes, epi- } \\
\text { lepsia; alterações do SNC. }\end{array}$ \\
\hline
\end{tabular}

* modificado de Watson $(1979)^{37}$

Os principais sinais e sintomas da intoxicação aguda e crônica pelo tolueno, n-hexano e cetonas (constituintes de colas) estão representados na Tabela 2.

A exposição aguda pode levar à morte devido a depressão respiratória. Também é comum a morte por asfixia em decorrência da fraqueza muscular (incluindo falência da musculatura respiratória) ou pela aspiração do próprio vômito $18,20,37,38$.

\section{DEPENDÊNCIA}

Parece haver um consenso entre os autores quanto ao desenvolvimento de tolerância e dependência psíquica em decorrência do uso prolongado de colas 14,16,24,28,37.

A frequêencia da exposição aos solventes pode influir no aparecimento da tolerância. Segundo Watson (1977) ${ }^{37}$, se a inalação é semanal, a tolerância já se instala após 3 semanas.

A dependência psíquica parece depender mais da intensidade da exposição do que do tipo de solvente. Com a descontinuidade no uso podem surgir a ansiedade e a depressão que levam o indivíduo a retornar ao uso dos solventes e a inalá-los quase sempre sozinho e não mais em grupo 27.

A dependência física - neuroadaptação não foi comprovada até o momento. Entretanto, são relatados alguns sinais e sintomas na supressão brusca dos solventes, que lembram uma síndrome de abstinência: calafrios, cefaléia, alucinações, delirium tremens ${ }^{27}$. 
TABELA 2

Principais efeitos nocivos decorrentes da intoxicação aguda e crônica pelo tolueno, $\mathbf{n}$-hexano e cetonas.

\begin{tabular}{|c|c|c|c|}
\hline $\begin{array}{l}\text { Intoxi- } \\
\text { cação }\end{array}$ & Tolueno & N-Hexano & Cetonas \\
\hline \multirow[t]{2}{*}{$\begin{array}{l}\text { A } \\
G \\
\text { U } \\
\text { D } \\
\text { A }\end{array}$} & $\begin{array}{l}\text { - SNC - alteração da consciên- } \\
\text { cia } \rightarrow \text { coma } \\
\quad \text { - alteraçōes neurológicas: } \\
\text { cefaléia, vertigens, problemas } \\
\text { motores. } \\
\text { - estado epiléptico }\end{array}$ & $\begin{array}{l}\text { - SNC - inconsciência } \\
\text { Irritação ocular e das } \\
\text { vias respiratórias supe- } \\
\text { riores. }\end{array}$ & $\begin{array}{l}\text { - SNC - cefaléia, sonolência, } \\
\text { confusão mental } \\
\quad \text { - depressão intensa: } \\
\text { coma e falência cardio res- } \\
\text { piratória. }\end{array}$ \\
\hline & $\begin{array}{l}\text { Alteraçōes hepáticas e renais } \\
\text { - Alteraçōes musculares e cardía- } \\
\text { cas } \\
\text { Alterações gastrintestinais: } \\
\text { náuseas e vốmitos }\end{array}$ & & $\begin{array}{l}\text { Irritação das mucosas, com } \\
\text { tosse, náusea e vômitos }\end{array}$ \\
\hline \multirow{3}{*}{$\begin{array}{l}\mathrm{C} \\
\mathrm{R} \\
\mathrm{O} \\
\mathrm{N} \\
\mathrm{I} \\
\mathrm{C}\end{array}$} & $\begin{array}{l}\text {-SNC - cefaléia crônica } \\
\text { - degeneração cerebelar }\end{array}$ & \multirow[t]{2}{*}{$\begin{array}{l}\text {-Pele - dermatose e si- } \\
\text { nais cu tâneos }\end{array}$} & \multirow{2}{*}{$\begin{array}{l}\text { Irritação ocular e das vias } \\
\text { respiratórias superiores; } \\
\text { gastralgias }\end{array}$} \\
\hline & $\begin{array}{l}\text { Encefalopatia } \\
\text { Permanente }\end{array}$ & & \\
\hline & - Polineuropatia & Polineuropatia & - Polineuropatia \\
\hline & $\begin{array}{l}\text { - Alterações hematológicas: } \\
\text { discrasias sangüíneas }\end{array}$ & & \\
\hline
\end{tabular}

SNC - Sistema nervoso central

\section{TRATAMENTO E PREVENÇÃO}

Embora a interrupção da inalação dos solventes seja bem tolerada por alguns individuos, vários tipos de tratamento são relatados na literatura. Em geral, o que se recomenda é psicoterapia em grupo ou individual associada à terapia ocupacional ou comportamental (associação do abuso a uma experiência desagradável, promovendo aversão ao uso destas substâncias) 2,4,24. A utilização de técnicas terapêuticas para com o usuário e com os familiares é uma tentativa louvável de forçar a alteração da estrutura familiar, incentivar a cooperação entre os membros, modificando o meio onde o adolescente vive.

É improvável que qualquer legislação limite, de maneira geral, o abuso de solventes devido ao grande número de substâncias envolvidas e à ampla utilização industrial e doméstica dos produtos que os contêm.

Em algumas cidades americanas restringiu-se a venda de colas a menores, o que só os incentivou a encontrar meios para burlar esta medida, quer solicitando a pessoas mais velhas para efetuarem a compra, quer roubando $4,25,28$.
Outra alternativa discutida é a adição às colas e outros produtos, de substâncias de odor desagradável como o óleo de mostarda e outras que provocam náuseas quando inaladas 2 .

A estratégia aparentemente mais adequada é a implantação de um programa educacional em nivel escolar para alertar os adolescentes quanto aos riscos deste uso. Alguns consideram que este alerta possa ser pernicioso por despertar a curiosidade de outros jovens ainda não usuários e aconselham que estas informações sejam fornecidas pelos próprios pais 4,28 .

Todavia, como já foi mencionado, a maioria dos usuários é proveniente de lares desfeitos ou desajustados onde não há clima adequado para discutir estes assuntos. Assim sendo, um programa de prevenção adequado deve enfocar o problema nos aspectos sócio-político-econômico, procurando aliar aos programas educacionais uma melhoria nas condições de vida desta classe social. O trabalho conjunto - governo, pais e educadores - poderá obter maior êxito nesse aspecto de que o trabalho individual de qualquer um deles. 
PEDROZO, M. de F.M. \& SIQUEIRA, M.E.P.B. de. [Glue solvents: abuse and harmful effects on health]. Rev. Saúde públ., S. Paulo, 23:336-40, 1989.

ABSTRACT: In Brazil, glue sniffing has been of growing concein due to the increasing number of abusers, most of them children and teen-agers of low socio-economic level. This review article presents some aspects of this kind of abuse, with special emphasis on the deleterious effects of acute and/or chronic solvent inhalation (glue-sniffing).

KEYWORDS: Substance abuse occurrence. Solvents, poisoning.

\section{REFERÊNCIAS BIBLIOGRÁFICAS}

1. ALLISON, W.M. \& JERROM, D.W.A. Glue sniffing: a pilot sludy of the cognitive effects of long-term use. Int. J. Addict, , 19: 453-8, 1984.

2. BARNES, G.E. Solvent abuse: a review. Int. J. Addict., 14: 1-26. 1975.

3. BASS, M. Sudden sniffing death. J. Amer. med. Ass., 212: $2075,1970$.

4. BLACK, D. Glue sniffing. Arch. Dis. Child., 57: 893-4, 1982.

5:BOWERS, A.J. \& SAGE, L.R. Solvent abuse in adolescents: the who? What? Why? Child Care Hith Dev., 9: 169-78, 1983.

6. BRUCKNER, J.V. \& PETERSON, R.G. Evaluation of Toluene and acetone inhálant abuse. Toxicol. Appl. Pharmacol., 61: 27-38, 1981.

7. CARLINI-CONTRIM, B. \& CARLINI. E.A. O consumo de solventes e outras drogas em crianças e adolescentes de baixa renda na cidade de São Paulo. Parte 1: Estudantes de primeiro e segundo graus da rede estadual. Revisia ABP-APAL, S. Paulo, 9(2): 49-58, 1987.

8. CARLINI-CONTRIM, B. \& CARLINI, E.A. O consumo de solventes e outras drogas em crianças e adolescentes de baixa renda na Grande Sào Paulo. Parte II: Meninos de rua e menores internados. Revista ABPAPAL, S. Paulo, 9:(2) 69-77, 1987.

9. CLEMENTS, J.E. Environmental and behavioral aspects of glue sniffing in a poputation of emotionally disturbed adolescents. Int. J. Addict., 13: 129-34, 1978.

10. COMSTOCK, B.S. A review of psychological measures relevant to central nervous system toxicity, with specific reference to solvent inhalation. Clin. Toxicol., 11: 317-24, 1977.

11. COOPER, R. et al. Neurophysicological signs of brain damage due to glue sniffing. Electroencephalogr. Clin. Neurophysiol., 60: 23-6, 1985.

12. COSTA, M.J.T.O. el al. Investigação sobre farmacodependência na população escolar da cidade de São Paulo.IMESC Ilus., S. Paulo, 2(3), 1979.

13. DE CAPRIO, A.P. et al. Neurotoxicity and protent bunding of 2.5 hexadione. Toxicol Appl. Pharmacol., 68: 297-307, 1983.

14. DEVATHASAN, G, et al. Complication of chronic glue (toluene) abuse in adolescents. Aust. N.Z.J. Med., 14:39-43, 1984.

15. EDWARDS, T.R. Solvent abuse. N.Z. med. J., 95: 879,1982

16. GARRIOT, T.J. Death from inhalant abuse: toxicological and pathological evaluation of 34 cases. Clin. Toxicol., 16:305-15, 1980.

17. GELLER, 1. et al. Toluene inhalation and anxiolytic activity: possible sinergism with DPZ. Pharmacol. Blochem. Behav., 19:899-903, 1983.

18. HAciUtNOER, J.M. \& FURON, D. Toxtcrstogte el hyglene mdustrelles. Parrs, I echmque et Documenta 1611. 1983, ․ 10, D. 91-198.

14. HAYDEN, JW. et al. The clinical toxicology of sol- vent abuse. Clin. Toxicol., 9:169-84, 1976.

20. HIBELE, B. \& JONSSON, C. Alcohol, drug, tabacco and sniffing practices among swedish school children 1971-1974. Brit. J. Addict., 72:117-27, 1977.

21. KORMAN, M. et al. Neuropsychological effects of abuse of inhalants. Percept. Mot. Skills, 53: 547-53, 1981.

22. KROEGER, M. et al. Recurrent urinary calculi associated with toluene sniffing. J. Urol., 123:89-91, 1980.

23. LECOMTE, D. et al. Complications pulmonaires de "sniffeurs". Rev. fr. Mal. respir., 11:713-7, 1983.

24. LOWENSTEIN, L.F. Glue sniffing: back ground features and treatment by aversion methods and group therapy. Practitioner, 226:1113-6, 1982.

25. LOWENSTEIN, L.F. Recent research into glue sniffingextents of the problem, its repercussions and treatment approaches. Int. J. soc. Psychiatr., 31:93-7, 1985.

26. MEDINA-MOURA, M.E. \& CASTRO, M.E. El uso de inhalants en Mexico. Salud ment., Mexico, 7(1): 13-8, 1984.

27. MOSS, A.H. et al. Fanconi's syndrome and Distal Renal tubular acidosis after glue sniffing. Ann. intern. Med., 92:69-70, 1980.

28. NATIONAL INSTITUTE ON DRUGS ABUSE. Voluntary inhalation of industrial solvents. Rockville, U.S. Department of Health, Education and Welfare, 1978.

29. O'CONNOR, D.J. A profile of solvent abuse in school children. J. Child. Psychol. Psychiatr., 20:365-8, 1975.

30. PADILLA, E.R. Inhalant, marijuana and alcohol abuse among barrio children and adolescents. Int. J. Addict., 14: 945-64, 1975.

31. PARKER, M.J. et al. Glue sniffing and cerebral infactions. Arch. Dis. Child., 59: 675-7, 1984.

32. STREICHER, H.Z. et al. Syndromes of toluene sniffing in adults. Ann. intern. Med., 94: 758-62, 1984.

33. SUWAKI, H. A follow up study of adolescents glue sniffers in Japan. Brit. J. Addict., 78: 409-13, 1982.

34. VIADER, F. et al. Polinevrite toxique chez un Iravailleur du plastique. Nouv. Presse med., 9: 1813-4, 1978.

35. WAKAMATSU, C.T. Contribuição ao estudo da exposição profissional ao benzeno em trabalhadores de indústrias de calçados, São Paulo. São Paulo, 1976. [Dissertação de Mestrado - Faculdade de Saúde Pública da USP].

36. WATSON, J.M. Glue sniffing in profile. Practitioner, 218: 255-9, 1977.

37. WATSON, J.M. Morbidity and mortality statistics on solvent abuse. Med. Scl. Law., 19: 247.52, 1979.

38. WATSON. J.M. Solvents abuse and adolescents. Practitioner, 228: 487-9, 1984.

Recebido para publicação em 1/12/1988 Reapresentado em 26/6/1989

Aprovado para publicaça em s/7/1989 